\title{
Frações da parede celular e digestibilidade in vitro da matéria seca de três genótipos de girassol ensilados com aditivos
}

[In vitro dry matter and cell wall fractions of three genotypes of sunflower ensiled with additives]

\author{
P.P. Porto ${ }^{1}$, E.O.S. Saliba ${ }^{2 *}$, L.C. Gonçalves ${ }^{2}$, N.M. Rodriguez ${ }^{2}$, I. Borges ${ }^{2}$, \\ A.L.C.C. Borges ${ }^{2}$, J.A.S. Rodrigues ${ }^{3}$, G.H.F. Ibrahim $^{2}$ \\ ${ }^{1}$ Universidade de Brasília \\ ${ }^{2}$ Escola de Veterinária - UFMG \\ Caixa Postal 567 \\ 30123-170 - Belo Horizonte, MG \\ ${ }^{3}$ EMBRAPA Milho e Sorgo - Sete Lagoas, MG
}

\begin{abstract}
RESUMO
Ensilaram-se três genótipos de girassol (M734, Rumbosol 91 e variedade V2000), enriquecidos no material original com: $0,5 \%$ de uréia (U); $0,5 \%$ de carbonato de cálcio (CC); $0,5 \%$ de uréia mais $0,5 \%$ de carbonato de cálcio (U+CC); inoculante bacteriano comercial (IB) comercial e sem aditivo que serviu como silagem testemunha (T). Foram utilizados silos de laboratório de PVC, abertos com 1, 3, 5, 7, 14, 28 e 56 dias de ensilados, sendo determinados fibra em detergente neutro (FDN), fibra em detergente ácido (FDA), lignina e digestibilidade in vitro da matéria seca (DIVMS). As silagens de Rumbosol 91 apresentaram valores superiores aos dos genótipos V2000 e M734 nos dias de abertura para FDN, FDA e lignina. Os aditivos não promoveram alterações nos constituintes da parede celular. A silagem $T$ não apresentou diferenças entre os genótipos quanto à DIVMS no decorrer do processo fermentativo, sendo os valores do último dia de abertura (56) de 51,0\%, 49,1\% e 48,9\% de DIVMS para os genótipos M734, V2000 e Rumbosol 91, respectivamente. Não houve diferença entre as silagens com aditivos e a silagem testemunha com a evolução do processo fermentativo quanto à DIVMS. Os aditivos utilizados não melhoraram as silagens de girassol quanto às características avaliadas e, apesar de os genótipos apresentarem digestibilidade in vitro semelhantes, o Rumbosol 91 apresentou maiores teores de constituintes da parede celular.
\end{abstract}

Palavras-chave: girassol, silagem, aditivo

\begin{abstract}
Three sunflower genotypes (M 734, Rumbosol 91 and V2000 variety) enriched with 0.5\% of urea (U); $0.5 \%$ of calcium carbonate (CC); $0.5 \%$ of urea plus $0.5 \%$ of calcium carbonate $(U+C C)$; commercial bacterial inoculate (BI); and without any additive, used as control silage (T) were ensiled in PVC silos and opened after 1, 3, 5, 7, 14, 28 and 56 days to determine the neutral detergent fiber (NDF), acid detergent fiber (FDA), lignin and dry matter in vitro digestibility (DMIVD). The Rumbosol 91 genotype silage showed higher NDF, ADF and lignin than V2000 and M734 genotypes. The additives did not promote changes in the cell wall constituents. No statistical differences among silages of the genotypes for DMIVD were observed during the fermentative process. The DMIVD at 56 days were 51.0, 49.1 and 48.9\% for silage of M734, V2000 and Rumbosol 91 genotypes, respectively. No difference between silages with additives and control (T), during the fermentative process for DMIVD was observed. The
\end{abstract}

Recebido em 1 de dezembro de 2004

Aceito em 7 de novembro de 2005

*Autor para correspondência (corresponding author)

E-mail: saliba@vet.ufmg.br

Apoio financeiro: CAPES 
additives did not improve sunflower silages. The genotypes showed similar in vitro digestibility, and the Rumbosol 91 genotype showed high compound of cell wall constituent.

Keywords: sunflower, silage, additive.

\section{INTRODUÇ̃̃̃O}

O girassol é uma oleaginosa com maior resistência à seca, ao frio e ao calor do que as culturas de milho e sorgo, as quais são normalmente plantadas no Brasil com o intuito de produzir silagem. Mais recentemente, tem sido utilizado como opção após a cultura do verão nas regiões Sudeste e Centro-Oeste para a confecção de silagem.

A silagem de girassol apresenta alto valor energético; e o teor de proteína pode ser até $35 \%$ superior ao do milho, viabilizando o balanceamento de rações a custos mais baixos devido à economia com a suplementação protéica (Souza, 1998).

$\mathrm{O}$ uso de aditivos tem o intuito de melhorar a qualidade e a conservação das silagens, modulando sua fermentação ou agregando maior valor nutritivo. A uréia é indicada como aditivo por promover o incremento das frações nitrogenadas deficientes em algumas culturas usadas na alimentação animal, enquanto o carbonato de cálcio serve como fonte de cálcio em culturas deficientes, como é o caso do milho (McDonald et al., 1991). A utilização de inoculantes bacterianos visa à maior produção de ácido láctico a partir dos carboidratos solúveis, promovendo, assim, uma boa fermentação do material ensilado.

Este trabalho teve como objetivo avaliar, em condições de laboratório, os teores de fibra em detergente neutro, fibra em detergente ácido, lignina e a digestibilidade, in vitro, da matéria seca das silagens de três genótipos de girassol tratadas com uréia, carbonato de cálcio, uréia associada a carbonato de cálcio e um inoculante bacteriano comercial, em sete diferentes épocas de abertura dos silos.

\section{MATERIAL E MÉTODOS}

Foram plantados, colhidos e ensilados três genótipos de girassol (M 734, Rumbosol 91 e a variedade V2000) nas dependências da
EMBRAPA Milho e Sorgo, no município de Sete Lagoas, MG.

Imediatamente após o corte, a forragem fresca foi enriquecida com os seguintes aditivos: $0,5 \%$ de uréia (U), $0,5 \%$ de carbonato de cálcio (CC), $0,5 \%$ de uréia mais $0,5 \%$ de carbonato de cálcio $(\mathrm{U}+\mathrm{CC})$ e inoculante bacteriano (IB) (Silobacsolução: $20 \mathrm{~g}$ em 201 de água; 21 solução/t forragem). Também foi ensilado material original sem aditivo que serviu como silagem testemunha (T).

Foram utilizados 210 silos de laboratório de PVC com $40 \mathrm{~cm}$ de comprimento e $10 \mathrm{~cm}$ de diâmetro.

Os silos foram abertos com 1, 3, 5, 7, 14, 28 e 56 dias de ensilados. O material retirado dos silos foi submetido a pré-secagem em estufa ventilada à $65^{\circ} \mathrm{C}$ por 72 horas e posterior moagem em partículas de $1 \mathrm{~mm}$ e armazenagem em frascos plásticos. Foram determinados os componentes da parede celular pelo método seqüencial utilizando $25 \mathrm{ml}$ de amilase termoestável ${ }^{1}$ a $1 \%$ adicionada no início da fervura, fibra em detergente neutro (FDN), fibra em detergente ácido (FDA), hemiceluloses, celulose e lignina (Van Soest, 1991) e digestibilidade in vitro da matéria seca (DIVMS) pela técnica descrita por Tilley e Terry (1963). Para a análise estatística, utilizou-se o pacote SAEG, VERSÃO 7.1. Para comparação de médias entre genótipos dentro de cada aditivo e entre aditivos dentro de cada genótipo e dia de abertura utilizou-se o teste SNK com 5\% de probabilidade. Empregou-se delineamento inteiramente ao acaso com duas repetições por tratamento, utilizando esquema fatorial $3 \times 5 \times 7$ ( 3 genótipos, 5 tratamentos e 7 dias de abertura).

\section{RESULTADOS E DISCUSSÃO}

Na Tab. 1 são apresentados os valores de FDN dos genótipos avaliados com os diferentes aditivos. As silagens do Rumbosol 91 apresentaram valores de FDN superiores aos dos

\footnotetext{
${ }^{1}$ Termamyl 1201 - Novo Nordisk Bioindustrial - Brasil
} 
genótipos V2000 e M734 nos diferentes dias de abertura avaliados. Nas silagens $T$, as concentrações de FDN foram em média de $53,6 \%, 46,7 \%$ e $46,6 \%$ para os genótipos Rumbosol 91, M734 e V2000, respectivamente. Não foi observada interação entre genótipos e aditivos. Tomich (1999) e Noguera (2001) encontraram valores de $47,6 \%$ e $49,5 \%$ para o Rumbosol 91, 50,5\% e 44,6\% para o M734 e $44,0 \%$ e $39,7 \%$ para as silagens de V2000, respectivamente. Observa-se variação para os teores de FDN de dados da literatura comparados aos do presente trabalho, os quais provavelmente estão relacionados a diferentes épocas de corte do material a ser ensilado, uma vez que o corte mais tardio das plantas acarreta aumento de sua fração fibrosa (Van Soest, 1994).
As silagens tratadas com $\mathrm{U}, \mathrm{CC}, \mathrm{U}+\mathrm{CC}$ e $\mathrm{IB}$ foram semelhantes à silagem $\mathrm{T}$ quanto aos teores de FDN dentro de cada genótipo nos diferentes dias de abertura. Os valores de FDN variaram de $49,0 \%$ a $56,3 \%$ nas silagens do Rumbosol 91, de $44,3 \%$ a $48,7 \%$ para as silagens do M734 e $41,6 \%$ a $47,2 \%$ para as silagens do V2000. Pires et al. (1998) trabalharam com 7,5\% de uréia em silagem de sorgo e observaram queda do teor de FDN, atribuída à provável ação da amônia liberada pela hidrólise da uréia sobre os constituintes da parede celular. Vieira (2001), ao estudar a adição de carbonato de cálcio em silagens de sorgo, verificou que o aditivo não interfere no teor de FDN, o que parece que o cálcio provavelmente não seja um nutriente limitante para as bactérias fibrolíticas.

Tabela 1. Concentrações de fibra em detergente neutro (\%MS) das silagens de girassol testemunha (T), tratadas com $0,5 \%$ de uréia (U), $0,5 \%$ de carbonato de cálcio (CC), 0,5\% de uréia associada a $0,5 \%$ de carbonato de cálcio (U+CC) e inoculante bacteriano (IB) em diferentes dias de abertura

\begin{tabular}{|c|c|c|c|c|c|c|c|c|}
\hline \multirow[b]{2}{*}{ Genótipo } & \multirow[b]{2}{*}{ Trat. } & \multicolumn{7}{|c|}{ Dias de abertura dos silos } \\
\hline & & 1 & 3 & 5 & 7 & 14 & 28 & 56 \\
\hline \multirow{5}{*}{ V21 } & $\mathrm{T}$ & $49,2 \mathrm{Aab} \beta$ & $47,5 \mathrm{Aab} \beta$ & $45,2 \mathrm{Ab} \beta$ & $51,2 \mathrm{Aa} \alpha$ & $47,4 \mathrm{Aab} \beta$ & $46,0 \mathrm{Aab} \beta$ & $44,8 \mathrm{Ab} \beta$ \\
\hline & $\mathrm{U}$ & $45,5 \mathrm{ABa} \beta$ & $43,2 \mathrm{ABa} \beta$ & $46,2 \mathrm{Aa \alpha}$ & $44,4 \mathrm{BCa} \beta$ & $47,2 \mathrm{Aa} \alpha$ & $43,2 \mathrm{Aa} \beta$ & $44,3 \mathrm{Aa} \beta$ \\
\hline & $\mathrm{CC}$ & $47,2 \mathrm{ABa} \beta$ & $45,1 \mathrm{ABa} \beta$ & $46,4 \mathrm{Aa} \beta$ & $45,8 \mathrm{BCa} \beta$ & $45,2 \mathrm{Aa} \beta$ & $45,0 \mathrm{Aa} \beta$ & $43,9 \mathrm{Aa} \beta$ \\
\hline & $\mathrm{U}+\mathrm{CC}$ & $42,9 \mathrm{Ba} \beta$ & $41,6 \mathrm{Ba} \beta$ & $44,6 \mathrm{Aa} \beta$ & $42,9 \mathrm{Ca} \lambda$ & $45,4 \mathrm{Aa} \beta$ & $43,5 \mathrm{Aa} \beta$ & $44,8 \mathrm{Aa} \beta$ \\
\hline & IB & $46,2 \mathrm{ABa} \beta$ & $44,8 \mathrm{ABa} \beta$ & $45,7 \mathrm{Aa} \beta$ & $48,2 \mathrm{ABa}$ & $45,6 \mathrm{Aa} \beta$ & $45,0 \mathrm{Aa} \beta$ & $43,9 \mathrm{Aa} \beta$ \\
\hline \multirow{5}{*}{ R91 } & $\mathrm{T}$ & $55,5 \mathrm{Aa} \alpha$ & $56,0 \mathrm{Aa} \alpha$ & $52,7 \mathrm{Aa \alpha}$ & $53,5 \mathrm{Aa} \alpha$ & $51,4 \mathrm{Aa} \alpha$ & $54,3 \mathrm{Aa} \alpha$ & $52,4 \mathrm{Aa} \alpha$ \\
\hline & $\mathrm{U}$ & $52,0 \mathrm{Aa} \alpha$ & $51,3 \mathrm{Aa} \alpha$ & $50,5 \mathrm{Aa} \alpha$ & $50,5 \mathrm{Aa} \alpha$ & $49,0 \mathrm{Aa} \alpha$ & $52,6 \mathrm{Aa} \alpha$ & $54,2 \mathrm{Aa} \alpha$ \\
\hline & $\mathrm{CC}$ & $54,2 \mathrm{Aa} \alpha$ & $54,3 \mathrm{Aa} \alpha$ & $53,8 \mathrm{Aa} \alpha$ & $52,6 \mathrm{Aa} \alpha$ & $53,1 \mathrm{Aa} \alpha$ & $54,4 \mathrm{Aa} \alpha$ & $53,9 \mathrm{Aa} \alpha$ \\
\hline & $\mathrm{U}+\mathrm{CC}$ & $54,4 \mathrm{Aa} \alpha$ & $54,7 \mathrm{Aa} \alpha$ & $54,1 \mathrm{Aa} \alpha$ & $54,8 \mathrm{Aa} \alpha$ & $53,4 \mathrm{Aa} \alpha$ & $56,3 \mathrm{Aa} \alpha$ & $56,3 \mathrm{Aa} \alpha$ \\
\hline & IB & $53,1 \mathrm{Aa} \alpha$ & $53,3 \mathrm{Aa} \alpha$ & $51,6 \mathrm{Aa} \alpha$ & $52,8 \mathrm{Aa} \alpha$ & $52,1 \mathrm{Aa} \alpha$ & $52,5 \mathrm{Aa} \alpha$ & $51,9 \mathrm{Aa} \alpha$ \\
\hline \multirow{5}{*}{ M71 } & $\mathrm{T}$ & $57,6 \mathrm{Aa} \alpha$ & $46,4 \mathrm{Bb} \beta$ & $49,2 \mathrm{Ab} \alpha$ & $46,8 \mathrm{Ab} \beta$ & $44,8 \mathrm{Ab} \beta$ & $47,0 \mathrm{Ab} \beta$ & $46,4 \mathrm{Ab} \beta$ \\
\hline & $\mathrm{U}$ & $47,5 \mathrm{Ba} \beta$ & $44,3 \mathrm{Ba} \beta$ & $47,0 \mathrm{Aa} \alpha$ & $47,3 \mathrm{Aa} \alpha \beta$ & $46,0 \mathrm{Aa} \alpha$ & $45,0 \mathrm{Aa} \beta$ & $46,4 \mathrm{Aa} \beta$ \\
\hline & $\mathrm{CC}$ & $48,7 \mathrm{Ba} \beta$ & $47,0 \mathrm{Ba} \beta$ & $46,9 \mathrm{Aa} \beta$ & $46,4 \mathrm{Aa} \beta$ & $46,6 \mathrm{Aa} \beta$ & $45,5 \mathrm{Aa} \beta$ & $45,2 \mathrm{Aa} \beta$ \\
\hline & $\mathrm{U}+\mathrm{CC}$ & $44,0 \mathrm{Ba} \beta$ & $44,6 \mathrm{Ba} \beta$ & $46,3 \mathrm{Aa} \beta$ & $46,7 \mathrm{Aa} \beta$ & $45,8 \mathrm{Aa} \beta$ & $43,6 \mathrm{Aa} \beta$ & $44,6 \mathrm{Aa} \beta$ \\
\hline & IB & $48,1 \mathrm{Ba} \beta$ & $50,9 \mathrm{Aa} \alpha$ & $47,8 \mathrm{Aa} \beta$ & $50,4 \mathrm{Aa} \alpha \beta$ & $47,7 \mathrm{Aa} \beta$ & $47,8 \mathrm{Aa} \beta$ & $46,4 \mathrm{Aa} \beta$ \\
\hline
\end{tabular}

Valores seguidos por letras maiúsculas distintas na mesma coluna, dentro de um genótipo, diferem entre si (P<0,05; efeito aditivo); Valores seguidos por letras minúsculas distintas na mesma linha diferem entre si ( $\mathrm{P}<0,05$; efeito abertura);

Valores seguidos por letras gregas distintas na mesma coluna diferem entre si ( $\mathrm{P}<0,05$; efeito de genótipo e aditivos);

${ }^{1} \mathrm{~V} 2=\mathrm{V} 2000 ; \mathrm{R} 9=$ Rumbosol 91; M7 = M734. CV = 3,898. Teste SNK $(\mathrm{P}<0,05)$.

A utilização de IB como aditivo não resultou em mudanças nos teores de FDN das silagens, demonstrando que, provavelmente, há afinidade dos microrganismos presentes no inoculante por substratos mais solúveis do que os existentes na parede celular. Isepon et al. (2000) avaliaram silagem de girassol com esse aditivo e não observaram diferenças nos teores de FDN entre a silagem controle $(47,2 \%)$ e a inoculada $(48,6 \%)$.

Os valores de FDA (Tab. 2) na silagem $T$ do Rumbosol 91 foram superiores quando comparados aos das silagens $\mathrm{T}$ dos genótipos V2000 e M734. Os resultados estão de acordo com os encontrados por Tomich (1999), que observou variação de $28,9 \%$ a $40,6 \%$ de FDA.

A adição de $\mathrm{U}, \mathrm{CC}$ e $\mathrm{U}+\mathrm{CC}$ não resultou em alterações na concentração de FDA, sendo os valores das silagens com aditivos semelhantes aos da silagem do tratamento $\mathrm{T}$ durante $\mathrm{o}$ processo fermentativo. O mesmo foi verificado entre os três tratamentos durante a fermentação. 
Tabela 2. Concentrações de fibra em detergente ácido (\%MS) das silagens de girassol testemunha (T), tratadas com $0,5 \%$ de uréia $(\mathrm{U}), 0,5 \%$ de carbonato de cálcio $(\mathrm{CC}), 0,5 \%$ de uréia associada a $0,5 \%$ de carbonato de cálcio (U+CC) e inoculante bacteriano (IB) em diferentes dias de abertura

\begin{tabular}{|c|c|c|c|c|c|c|c|c|}
\hline \multirow[b]{2}{*}{ Genótipo } & \multirow[b]{2}{*}{ Trat } & \multicolumn{7}{|c|}{ Dias de abertura dos silos } \\
\hline & & 1 & 3 & 5 & 7 & 14 & 28 & 56 \\
\hline \multirow{5}{*}{$\mathrm{V} 21$} & $\mathrm{~T}$ & $37,6 \mathrm{Aab} \beta$ & $34,9 \mathrm{Ab} \beta$ & $34,2 \mathrm{Ab} \beta$ & $39,8 \mathrm{Aa} \alpha$ & $35,2 \mathrm{Ab} \beta$ & $35,2 \mathrm{Ab} \beta$ & $35,5 \mathrm{Ab} \beta$ \\
\hline & $\mathrm{U}$ & $34,7 \mathrm{ABa} \beta$ & $32,0 \mathrm{ABa} \beta$ & $34,5 \mathrm{Aa} \beta$ & $33,4 \mathrm{Ba} \beta$ & $35,7 \mathrm{Aa} \alpha$ & $33,2 \mathrm{Aa} \beta$ & $35,3 \mathrm{Aa} \beta$ \\
\hline & $\mathrm{CC}$ & $34,8 \mathrm{ABa} \beta$ & $33,3 \mathrm{ABa} \beta$ & $34,4 \mathrm{Aa} \beta$ & $34,1 \mathrm{Ba} \beta$ & $33,3 \mathrm{Aa} \beta$ & $34,0 \mathrm{Aa} \beta$ & $35,0 \mathrm{Aa} \beta$ \\
\hline & $\mathrm{U}+\mathrm{CC}$ & $32,3 \mathrm{Ba} \beta$ & $30,6 \mathrm{Ba} \lambda$ & $32,8 \mathrm{Aa} \beta$ & $31,8 \mathrm{Ba} \beta$ & $33,5 \mathrm{Aa} \beta$ & $32,6 \mathrm{Aa} \beta$ & $33,6 \mathrm{Aa} \beta$ \\
\hline & IB & $33,8 \mathrm{ABa} \beta$ & $33,6 \mathrm{ABa} \beta$ & $33,1 \mathrm{Aa} \lambda$ & $33,8 \mathrm{Ba} \lambda$ & $33,4 \mathrm{Aa} \lambda$ & $33,6 \mathrm{Aa} \beta$ & $33,5 \mathrm{Aa} \beta$ \\
\hline \multirow{5}{*}{ R91 } & $\mathrm{T}$ & $42,3 \mathrm{Aa \alpha}$ & $42,8 \mathrm{Aa \alpha}$ & $41,2 \mathrm{Aa} \alpha$ & $42,1 \mathrm{Aa} \alpha$ & $39,3 \mathrm{ABa} \alpha$ & $42,2 \mathrm{Aa} \alpha$ & $41,3 \mathrm{Aa} \alpha$ \\
\hline & $\mathrm{U}$ & $39,5 \mathrm{Aa \alpha}$ & $40,1 \mathrm{Aa} \alpha$ & $38,4 \mathrm{Aa} \alpha$ & $39,3 \mathrm{Aa} \alpha$ & $37,4 \mathrm{Ba} \alpha$ & $40,4 \mathrm{Aa} \alpha$ & $41,8 \mathrm{Aa} \alpha$ \\
\hline & $\mathrm{CC}$ & $41,4 \mathrm{Aa} \alpha$ & $42,2 \mathrm{Aa} \alpha$ & $41,5 \mathrm{Aa} \alpha$ & $40,8 \mathrm{Aa} \alpha$ & $41,5 \mathrm{ABa} \alpha$ & $41,4 \mathrm{Aa} \alpha$ & $42,0 \mathrm{Aa} \alpha$ \\
\hline & $\mathrm{U}+\mathrm{CC}$ & 41,7Aa $\alpha$ & $42,8 \mathrm{Aa} \alpha$ & 41,9Аa $\alpha$ & $42,1 \mathrm{Aa} \alpha$ & 41,9Aa $\alpha$ & $43,8 \mathrm{Aa} \alpha$ & $43,6 \mathrm{Aa} \alpha$ \\
\hline & IB & $41,1 \mathrm{Aa} \alpha$ & $41,1 \mathrm{Aa} \alpha$ & $40,3 \mathrm{Aa} \alpha$ & $40,6 \mathrm{Aa} \alpha$ & $40,3 \mathrm{ABa} \alpha$ & $40,4 \mathrm{Aa} \alpha$ & $40,8 \mathrm{Aa} \alpha$ \\
\hline \multirow{5}{*}{ M71 } & $\mathrm{T}$ & $42,9 \mathrm{Aa} \alpha$ & $35,1 \mathrm{ABb} \beta$ & $35,6 \mathrm{Ab} \beta$ & $34,1 \mathrm{Ab} \beta$ & $34,2 \mathrm{Ab} \beta$ & $35,2 \mathrm{Ab} \beta$ & $34,2 \mathrm{Ab} \beta$ \\
\hline & $\mathrm{U}$ & $34,9 \mathrm{Ba} \beta$ & $33,1 \mathrm{Ba} \beta$ & $35,3 \mathrm{Aa} \beta$ & $35,4 \mathrm{Aa} \beta$ & $35,3 \mathrm{Aa} \alpha$ & $34,0 \mathrm{Aa} \beta$ & $36,9 \mathrm{Aa} \beta$ \\
\hline & $\mathrm{CC}$ & $35,7 \mathrm{Ba} \beta$ & $35,7 \mathrm{ABa} \beta$ & $35,0 \mathrm{Aa} \beta$ & $34,6 \mathrm{Aa} \beta$ & $35,5 \mathrm{Aa} \beta$ & $34,9 \mathrm{Aa} \beta$ & $35,3 \mathrm{Aa} \beta$ \\
\hline & $\mathrm{U}+\mathrm{CC}$ & $33,2 \mathrm{Ba} \beta$ & $33,7 \mathrm{Ba} \beta$ & $34,4 \mathrm{Aa} \beta$ & $33,6 \mathrm{Aa} \beta$ & $34,1 \mathrm{Aa} \beta$ & $33,5 \mathrm{Aa} \beta$ & $34,5 \mathrm{Aa} \beta$ \\
\hline & IB & $36,8 \mathrm{Ba} \beta$ & $38,3 \mathrm{Aa} \alpha$ & $36,2 \mathrm{Aa} \beta$ & $37,1 \mathrm{Aa} \beta$ & $36,5 \mathrm{Aa} \beta$ & $36,3 \mathrm{Aa} \beta$ & $36,0 \mathrm{Aa} \beta$ \\
\hline
\end{tabular}

Valores seguidos por letras maiúsculas distintas na mesma coluna, dentro de um genótipo, diferem entre si ( $\mathrm{P}<0,05$; efeito aditivo);

Valores seguidos por letras minúsculas distintas na mesma linha diferem entre si $(\mathrm{P}<0,05$; efeito abertura);

Valores seguidos por letras gregas distintas na mesma coluna diferem entre si $(\mathrm{P}<0,05$; efeito de genótipo e aditivos);

${ }^{1} \mathrm{~V} 2$ = V2000; R9 = Rumbosol 91; M7 = M734. CV = 4,233. Teste SNK $(\mathrm{P}<0,05)$.

A silagem com IB não apresentou alteração em relação aos teores de FDA quando comparada com a silagem $\mathrm{T}$ em, praticamente, todos os genótipos e no decorrer do processo fermentativo. Este resultado é semelhante aos de Meeske et al. (1993) e Higginbotham et al. (1998), que trabalharam com diferentes inoculantes bacterianos em silagem de sorgo e de milho, respectivamente. De maneira geral, os teores de FDA apenas foram afetados pelo genótipo, não se observou efeito da interação genótipo $\times$ aditivo.

Os menores valores de hemicelulose nas silagens dos genótipos Rumbosol 91, M734 e V2000 foram $11,0 \% ; 9,4 \%$; e $8,8 \%$, respectivamente. Em quase $60 \%$ dos dados os resultados entre os genótipos se equivaleram (Tab. 3).

A adição de $U$ resultou em diminuição significativa da hemicelulose na silagem do genótipo M734, enquanto que na do V2000 e do Rumbosol 91 praticamente não ocorreram alterações. Vieira (2001) não observou reduções nos teores de hemiceluloses ao trabalhar com silagens de sorgo com o mesmo aditivo.

O uso de $\mathrm{CC}$ no $56^{\circ}$ dia de abertura resultou na diminuição da hemicelulose da silagem do genótipo V2000 e diminuição progressiva no $\mathrm{M} 734$, enquanto $\mathrm{U}+\mathrm{CC}$ não teve efeito em nenhuma das silagens avaliadas. A silagem do V2000 com CC apresentou queda significativa de $12,4 \%$ para $8,8 \%$ de hemicelulose entre o primeiro dia de abertura e o $56^{\circ}$ dia de fermentação, enquanto a silagem do M734 decresceu de $12,9 \%$ para $9,9 \%$.

Entretanto, o que chama a atenção para os efeitos dos aditivos nas silagens, quanto à concentração de hemicelulose, foi a diferença significativa encontrada entre a silagem $\mathrm{T}$ e todos os aditivos utilizados neste experimento para o dia 56 no genótipo M734.

De acordo com Muck (1988) e McDonald et al. (1991), podem ocorrer perdas de hemicelulose nas silagens, pois essas servirão como principal fonte adicional de substrato para a fermentação ácido láctica, sendo isso dependente do estádio de crescimento bacteriano e do conteúdo de MS da forrageira ensilada. A hemicelulose é formada por vários açúcares, os quais podem sofrer fermentação se passarem por algum tipo de processamento físico ou químico que venha a romper os polímeros e os exponham ao meio. Porém, as diferenças observadas nos teores de hemicelulose não acarretaram alterações nos teores de FDN (Tab.1), como poderia ter acontecido já que as hemiceluloses fazem parte da parede celular. 
Frações da parede celular...

Tabela 3 - Concentrações de hemicelulose (\%MS) das silagens de girassol testemunha (T), tratadas com $0,5 \%$ de uréia $(\mathrm{U}), 0,5 \%$ de carbonato de cálcio $(\mathrm{CC}), 0,5 \%$ de uréia associada a $0,5 \%$ de carbonato de cálcio (U+CC) e inoculante bacteriano (IB) em diferentes dias de abertura

\begin{tabular}{|c|c|c|c|c|c|c|c|c|}
\hline \multirow[b]{2}{*}{ Genótipo } & \multirow[b]{2}{*}{ Trat. } & \multicolumn{7}{|c|}{ Dias de abertura dos silos } \\
\hline & & 1 & 3 & 5 & 7 & 14 & 28 & 56 \\
\hline \multirow{5}{*}{$\mathrm{V} 21$} & $\mathrm{~T}$ & $11,5 \mathrm{Aa} \lambda$ & $12,5 \mathrm{Aa} \alpha \beta$ & $11,0 \mathrm{Aab} \beta$ & $11,3 \mathrm{Ba \alpha}$ & $12,1 \mathrm{Aa} \alpha$ & $10,8 \mathrm{Aab} \alpha$ & $9,2 \mathrm{Bb} \beta$ \\
\hline & $\mathrm{U}$ & $10,8 \mathrm{Aab} \beta$ & $11,1 \mathrm{Aab} \alpha$ & $11,6 \mathrm{Aa} \alpha$ & $10,9 \mathrm{Bab} \alpha$ & $11,4 \mathrm{Aa} \alpha$ & $9,9 \mathrm{Aab} \beta$ & $9,0 \mathrm{Bb} \beta$ \\
\hline & $\mathrm{CC}$ & $12,4 \mathrm{Aa} \alpha$ & $11,7 \mathrm{Aa} \alpha$ & $11,9 \mathrm{Aa} \alpha$ & $11,7 \mathrm{Ba} \alpha$ & $11,9 \mathrm{Aa} \alpha$ & $10,9 \mathrm{Aa} \beta$ & $8,8 \mathrm{Bb} \beta$ \\
\hline & $\mathrm{U}+\mathrm{CC}$ & $10,5 \mathrm{Aa} \beta$ & $10,9 \mathrm{Aa} \alpha$ & $11,8 \mathrm{Aa} \alpha$ & $11,1 \mathrm{Ba} \beta$ & $11,9 \mathrm{Aa} \alpha$ & $10,8 \mathrm{Aa} \beta$ & $11,2 \mathrm{Aa} \beta$ \\
\hline & IB & $12,3 \mathrm{Ab} \alpha$ & $11,2 \mathrm{Ab} \alpha$ & $12,5 \mathrm{Ab} \alpha$ & $14,3 \mathrm{Aa} \alpha$ & $12,2 \mathrm{Ab} \alpha$ & $11,3 \mathrm{Ab} \alpha$ & $10,3 \mathrm{ABb} \alpha$ \\
\hline \multirow{5}{*}{ R91 } & $\mathrm{T}$ & $13,1 \mathrm{Aa} \beta$ & $13,1 \mathrm{Aa} \alpha$ & $11,5 \mathrm{Aa} \beta$ & $11,4 \mathrm{Aa} \alpha$ & $12,0 \mathrm{Aa} \alpha$ & $12,0 \mathrm{Aa} \alpha$ & $11,1 \mathrm{Aa} \alpha$ \\
\hline & $\mathrm{U}$ & $12,4 \mathrm{Aa} \alpha$ & $11,2 \mathrm{Aa} \alpha$ & $12,1 \mathrm{Aa} \alpha$ & $11,2 \mathrm{Aa} \alpha$ & $11,5 \mathrm{Aa} \alpha$ & $12,2 \mathrm{Aa} \alpha$ & $12,3 \mathrm{Aa} \alpha$ \\
\hline & $\mathrm{CC}$ & $12,7 \mathrm{Aa} \alpha$ & $12,1 \mathrm{Aa} \alpha$ & $12,3 \mathrm{Aa} \alpha$ & $11,8 \mathrm{Aa} \alpha$ & $11,6 \mathrm{Aa} \alpha$ & $13,0 \mathrm{Aa} \alpha$ & 11,9Aa $\alpha$ \\
\hline & $\mathrm{U}+\mathrm{CC}$ & $12,6 \mathrm{Aa} \alpha$ & 11,9Aa $\alpha$ & $12,1 \mathrm{Aa} \alpha$ & $12,6 \mathrm{Aa} \alpha$ & $11,4 \mathrm{Aa} \alpha$ & $12,5 \mathrm{Aa} \alpha$ & $12,7 \mathrm{Aa \alpha}$ \\
\hline & IB & $12,0 \mathrm{Aa} \alpha$ & $12,2 \mathrm{Aa} \alpha$ & $11,3 \mathrm{Aa} \alpha$ & $12,1 \mathrm{Aa} \beta$ & $11,8 \mathrm{Aa} \alpha$ & $12,1 \mathrm{Aa} \alpha$ & $11,0 \mathrm{Aa} \alpha$ \\
\hline \multirow{5}{*}{ M71 } & $\mathrm{T}$ & $14,7 \mathrm{Aa} \alpha$ & $11,2 \operatorname{Acd} \beta$ & $13,6 \mathrm{Aab} \alpha$ & $12,7 \mathrm{Abc} \alpha$ & $10,5 \operatorname{Ad} \alpha$ & $11,8 \mathrm{Abcd} \alpha$ & $12,1 \mathrm{Abcd} \alpha$ \\
\hline & $\mathrm{U}$ & $12,6 \mathrm{Ba} \alpha$ & $11,1 \mathrm{Aab} \alpha$ & $11,7 \mathrm{ABa} \alpha$ & $11,8 \mathrm{Aa} \alpha$ & $10,7 \mathrm{Aab} \alpha$ & $10,9 \mathrm{Aab} \alpha \beta$ & $9,4 \mathrm{Bb} \beta$ \\
\hline & $\mathrm{CC}$ & $12,9 \mathrm{Ba} \alpha$ & $11,2 \mathrm{Aab} \alpha$ & $11,8 \mathrm{Aab} \alpha$ & $11,8 \mathrm{Aab} \alpha$ & $11,0 \mathrm{Aab} \alpha$ & $10,5 \mathrm{Ab} \beta$ & $9,9 \mathrm{Bb} \beta$ \\
\hline & $\mathrm{U}+\mathrm{CC}$ & $10,8 \mathrm{Cb} \beta$ & $10,8 \mathrm{Ab} \alpha$ & $11,8 \mathrm{Bab} \alpha$ & $13,0 \mathrm{Aa} \alpha$ & $10,9 \mathrm{Ab} \alpha$ & $10,1 \mathrm{Ab} \beta$ & $10,0 \mathrm{Bb} \beta$ \\
\hline & IB & $11,3 \mathrm{BCab} \alpha$ & $12,6 \mathrm{Aa} \alpha$ & $11,5 \mathrm{ABab} \alpha$ & $13,3 \mathrm{Aa} \alpha \beta$ & $11,1 \mathrm{Aabb} \alpha$ & $11,4 \mathrm{Aab} \alpha$ & $10,3 \mathrm{Bb} \alpha$ \\
\hline
\end{tabular}

Valores seguidos por letras maiúsculas distintas na mesma coluna, dentro de um genótipo, diferem entre si ( $\mathrm{P}<0,05$; efeito aditivo); Valores seguidos por letras minúsculas distintas na mesma linha diferem entre si $(\mathrm{P}<0,05$; efeito abertura);

Valores seguidos por letras gregas distintas na mesma coluna diferem entre si $(\mathrm{P}<0,05$; efeito de genótipo e aditivos);

${ }^{1} \mathrm{~V} 2=\mathrm{V} 2000 ; \mathrm{R} 9=$ Rumbosol 91; M7 = M734. CV =6,762. Teste SNK $(\mathrm{P}<0,05)$.

Os resultados dos teores de celulose na Tab. 4 para as silagens $\mathrm{T}$ entre os genótipos variaram de $26,1 \%$ a $32,9 \%$. Diferenças referentes ao genótipo também foram observadas por Freire (2001) em silagens de cinco genótipos de girassol, sendo que os valores encontrados variaram de 23,6 a $29,6 \%$.

O avanço do processo fermentativo não acarretou alterações nos teores de celulose nas silagens $\mathrm{T}$ dos três genótipos, o mesmo foi observado para as silagens com os aditivos. De acordo com Van Soest (1994), o teor de celulose no silo durante o processo fermentativo é estável, sendo que a diminuição em seus teores pode ocorrer com a deterioração aeróbica. Entretanto, alguns trabalhos têm relatado possível utilização da celulose como substrato durante o processo fermentativo (Silva, 1997).

Em relação aos aditivos, foram observadas diferenças apenas para as silagens do genótipo V2000 nos dias de abertura 1, 3 e 7, e para o M734 nos dias 1, 3 e 5. Entretanto, somente o tratamento com $\mathrm{U}+\mathrm{CC}$ nos dias de abertura 1 e 3 e com inoculante no primeiro dia foram estatisticamente diferentes das silagens testemunhas. Segundo Tomich (1999), as dificuldades na obtenção da amostra de FDA proveniente do resíduo de FDN com conseqüente formação de partículas compactas que diminuíam a superfície de contato com o detergente ácido, de acordo com o método de análise utilizado, podem ser responsáveis por estas diferenças nos valores intermediários das silagens analisadas.

O Rumbosol 91 apresentou os maiores valores de lignina (Tab. 5), tanto no tratamento $\mathrm{T}$ quanto com aditivos, se comparados aos dos genótipos V2000 e M734. As menores concentrações de lignina foram: $7,9 \% ; 6,2 \%$; e $6,1 \%$, para Rumbosol 91, M734 e V2000, respectivamente.

Quanto aos efeitos de aditivo e dia de abertura sobre as porcentagens de lignina nas silagens, exceto o tratamento com IB para o dia de abertura 14 no M734 com 8,5\%, não houve diferença nessa variável para os tratamentos. Os resultados do tratamento com inoculante foram similares à silagem testemunha, confirmando os achados de Hunt et al. (1993) em silagens de milho.

A utilização de uréia não promoveu alteração no teor de lignina, Schingoethe et al. (1980), ao trabalharem com silagens de girassol, observaram diminuição desse composto na silagem tratada com uréia em relação à silagem controle. 
Tabela 4. Concentrações de celulose (\% MS) das silagens de girassol testemunha (T), tratadas com $0,5 \%$ de uréia $(\mathrm{U}), 0,5 \%$ de carbonato de cálcio $(\mathrm{CC}), 0,5 \%$ de uréia associada a $0,5 \%$ de carbonato de cálcio (U+CC) e inoculante bacteriano (IB) nos diferentes dias de abertura

\begin{tabular}{|c|c|c|c|c|c|c|c|c|}
\hline \multirow[b]{2}{*}{ Genótipo } & \multirow[b]{2}{*}{ Trat. } & \multicolumn{7}{|c|}{ Dias de abertura dos silos } \\
\hline & & 1 & 3 & 5 & 7 & 14 & 28 & 56 \\
\hline \multirow{5}{*}{ V21 } & $\mathrm{T}$ & $28,9 \mathrm{Aa} \beta$ & $27,7 \mathrm{Aa} \beta$ & $26,1 \mathrm{Aa} \beta$ & $29,3 \mathrm{Aa} \beta$ & $27,0 \mathrm{Aa} \beta$ & $26,8 \mathrm{Aa} \beta$ & $27,2 \mathrm{Aa} \beta$ \\
\hline & $\mathrm{U}$ & $26,4 \mathrm{ABa} \beta$ & $25,1 \mathrm{ABa} \beta$ & $26,2 \mathrm{Aa \alpha}$ & $25,8 \mathrm{Ba} \beta$ & $27,4 \mathrm{Aa \alpha}$ & $24,9 \mathrm{Aa} \beta$ & $26,6 \mathrm{Aa} \beta$ \\
\hline & $\mathrm{CC}$ & $26,6 \mathrm{ABa} \beta$ & $26,3 \mathrm{ABa} \beta$ & $26,9 \mathrm{Aa} \beta$ & $26,1 \mathrm{Ba} \beta$ & $25,5 \mathrm{Aa} \lambda$ & $26,5 \mathrm{Aa} \beta$ & $27,6 \mathrm{Aa} \beta$ \\
\hline & $\mathrm{U}+\mathrm{CC}$ & $24,3 \mathrm{Ba} \beta$ & $23,7 \mathrm{Ba} \lambda$ & $25,7 \mathrm{Aa} \beta$ & $24,1 \mathrm{Ba} \beta$ & $26,0 \mathrm{Aa} \beta$ & $25,4 \mathrm{Aa} \beta$ & $25,6 \mathrm{Aa} \beta$ \\
\hline & IB & $25,3 \mathrm{Ba} \lambda$ & $26,1 \mathrm{ABa} \beta$ & $25,5 \mathrm{Aa} \beta$ & $26,1 \mathrm{Ba} \beta$ & $25,7 \mathrm{Aa} \beta$ & $25,7 \mathrm{Aa} \beta$ & $26,1 \mathrm{Aa} \lambda$ \\
\hline \multirow{5}{*}{ R91 } & $\mathrm{T}$ & $32,3 \mathrm{Aa} \alpha$ & $32,9 \mathrm{Aa} \alpha$ & $31,2 \mathrm{Aa} \alpha$ & $32,6 \mathrm{Aa} \alpha$ & $30,5 \mathrm{Aa} \alpha$ & $32,7 \mathrm{Aa \alpha}$ & $32,4 \mathrm{Aa} \alpha$ \\
\hline & $\mathrm{U}$ & $30,3 \mathrm{Aa} \alpha$ & $30,4 \mathrm{Aa} \alpha$ & $29,2 \mathrm{Aa} \alpha$ & $30,4 \mathrm{Aa} \alpha$ & $28,9 \mathrm{Aa} \alpha$ & $31,2 \mathrm{Aa} \alpha$ & $32,6 \mathrm{Aa} \alpha$ \\
\hline & $\mathrm{CC}$ & $32,0 \mathrm{Aa} \alpha$ & $32,8 \mathrm{Aa} \alpha$ & $31,5 \mathrm{Aa} \alpha$ & $31,5 \mathrm{Aa} \alpha$ & $31,8 \mathrm{Aa} \alpha$ & $31,9 \mathrm{Aa} \alpha$ & $33,1 \mathrm{Aa} \alpha$ \\
\hline & $\mathrm{U}+\mathrm{CC}$ & $32,4 \mathrm{Aa} \alpha$ & $32,9 \mathrm{Aa} \alpha$ & $32,3 \mathrm{Aa} \alpha$ & $32,3 \mathrm{Aa} \alpha$ & $32,5 \mathrm{Aa} \alpha$ & $33,0 \mathrm{Aa} \alpha$ & $34,5 \mathrm{Aa} \alpha$ \\
\hline & IB & $31,9 \mathrm{Aa} \alpha$ & $31,2 \mathrm{Aa} \alpha$ & $30,3 \mathrm{Aa} \alpha$ & $31,1 \mathrm{Aa} \alpha$ & $30,3 \mathrm{Aa} \alpha$ & $31,0 \mathrm{Aa} \alpha$ & $32,0 \mathrm{Aa} \alpha$ \\
\hline \multirow{5}{*}{ M71 } & $\mathrm{T}$ & $32,8 \mathrm{Aa \alpha}$ & $27,8 \mathrm{ABb} \beta$ & $27,8 \mathrm{Ab} \beta$ & $26,1 \mathrm{Ab} \lambda$ & $27,4 \mathrm{Ab} \beta$ & $27,3 \mathrm{Ab} \beta$ & $27,2 \mathrm{Ab} \beta$ \\
\hline & $\mathrm{U}$ & $27,3 \mathrm{BCa} \beta$ & $26,2 \mathrm{Ba} \beta$ & $27,9 \mathrm{Aa} \alpha$ & $28,1 \mathrm{Aa} \alpha \beta$ & $28,2 \mathrm{Aa} \alpha$ & $26,9 \mathrm{Aa} \beta$ & $29,9 \mathrm{Aa} \alpha$ \\
\hline & $\mathrm{CC}$ & $27,1 \mathrm{BCa} \beta$ & $27,9 \mathrm{ABa} \beta$ & $23,8 \mathrm{Bb} \lambda$ & $26,7 \mathrm{Aa} \beta$ & $28,4 \mathrm{Aa} \beta$ & $27,6 \mathrm{Aa} \beta$ & $28,5 \mathrm{Aa} \beta$ \\
\hline & $\mathrm{U}+\mathrm{CC}$ & $24,7 \mathrm{Ca} \beta$ & $26,4 \mathrm{Ba} \beta$ & $27,1 \mathrm{Aa} \beta$ & $26,6 \mathrm{Aa} \beta$ & $27,5 \mathrm{Aa} \beta$ & $26,3 \mathrm{Aa} \beta$ & $27,7 \mathrm{Aa} \beta$ \\
\hline & IB & $28,6 \mathrm{Ba} \beta$ & $30,5 \mathrm{Aa} \alpha$ & $29,1 \mathrm{Aa} \alpha$ & $29,7 \mathrm{Aa} \alpha$ & $27,8 \mathrm{Aa} \alpha \beta$ & $28,6 \mathrm{Aa} \alpha$ & $29,3 \mathrm{Aa} \beta$ \\
\hline
\end{tabular}

Valores seguidos por letras maiúsculas distintas na mesma coluna, dentro de um genótipo, diferem entre si (P $<0,05$; efeito aditivo); Valores seguidos por letras minúsculas distintas na mesma linha diferem entre si $(\mathrm{P}<0,05$; efeito abertura);

Valores seguidos por letras gregas distintas na mesma coluna diferem entre si $(\mathrm{P}<0,05$; efeito de genótipo e aditivos);

${ }^{1} \mathrm{~V} 2$ = V2000; R9 = Rumbosol 91; M7 = M734. CV = 4,233. Teste SNK $(\mathrm{P}<0,05)$.

Tabela 5 - Concentrações de lignina (\% MS) das silagens de girassol testemunha (T), tratadas com 0,5\% de uréia (U), $0,5 \%$ de carbonato de cálcio (CC), 0,5\% de uréia associada a $0,5 \%$ de carbonato de cálcio $(\mathrm{U}+\mathrm{CC})$ e inoculante bacteriano (IB) nos diferentes dias de abertura

\begin{tabular}{|c|c|c|c|c|c|c|c|c|}
\hline \multirow[b]{2}{*}{ Genótipo } & \multirow[b]{2}{*}{ Trat } & \multicolumn{7}{|c|}{ Dias de abertura dos silos } \\
\hline & & 1 & 3 & 5 & 7 & 14 & 28 & 56 \\
\hline \multirow{5}{*}{ V21 } & $\mathrm{T}$ & $7,6 \mathrm{Ab} \beta$ & $7,1 \mathrm{Ab} \beta$ & $6,3 \mathrm{Ab} \beta$ & $9,2 \mathrm{Aa} \alpha$ & $7,5 \mathrm{Ab} \alpha$ & $7,6 \mathrm{Ab} \beta$ & $7,7 \mathrm{Ab} \alpha$ \\
\hline & $\mathrm{U}$ & $6,1 \mathrm{Ab} \beta$ & $6,1 \mathrm{Ab} \beta$ & $7,7 \mathrm{Aa} \beta$ & $7,6 \mathrm{Ba} \alpha \beta$ & $7,8 \mathrm{Aa} \alpha$ & $7,0 \mathrm{Aab} \beta$ & $8,2 \mathrm{Aa} \alpha$ \\
\hline & $\mathrm{CC}$ & $7,6 \mathrm{Aa} \alpha$ & $6,5 \mathrm{Aa} \beta$ & $7,1 \mathrm{Aa} \beta$ & $7,6 \mathrm{Ba} \beta$ & $7,1 \mathrm{Aa} \beta$ & $7,4 \mathrm{Aa} \beta$ & $7,3 \mathrm{Aa} \beta$ \\
\hline & $\mathrm{U}+\mathrm{CC}$ & $7,3 \mathrm{Aa} \beta$ & $6,2 \mathrm{Aa} \beta$ & $6,9 \mathrm{Aa} \beta$ & $7,2 \mathrm{Ba} \beta$ & $7,1 \mathrm{Aa} \beta$ & $7,1 \mathrm{Aa} \beta$ & 7,5Aa $\alpha$ \\
\hline & IB & $7,2 \mathrm{Aa} \alpha$ & $6,5 \mathrm{Aa} \beta$ & $7,3 \mathrm{Aa} \beta$ & $7,5 \mathrm{Ba} \beta$ & $7,4 \mathrm{Aa} \beta$ & $7,7 \mathrm{Aa} \alpha \beta$ & $7,0 \mathrm{Aa} \beta$ \\
\hline \multirow{5}{*}{ R91 } & $\mathrm{T}$ & $9,1 \mathrm{Aa} \alpha$ & $9,1 \mathrm{Aa} \alpha$ & $9,4 \mathrm{Aa} \alpha$ & $8,9 \mathrm{Aa} \alpha$ & $8,3 \mathrm{Aa} \alpha$ & $8,9 \mathrm{Aa} \alpha$ & $7,9 \mathrm{Aa} \alpha$ \\
\hline & $\mathrm{U}$ & $8,7 \mathrm{Aa} \alpha$ & $9,1 \mathrm{Aa} \alpha$ & $8,9 \mathrm{Aa} \alpha$ & $8,5 \mathrm{Aa} \alpha$ & 7,9Aa $\alpha$ & $8,5 \mathrm{Aa} \alpha$ & $8,4 \mathrm{Aa} \alpha$ \\
\hline & $\mathrm{CC}$ & $8,7 \mathrm{Aa} \alpha$ & $8,9 \mathrm{Aa} \alpha$ & $9,5 \mathrm{Aa} \alpha$ & $8,9 \mathrm{Aa} \alpha$ & $9,1 \mathrm{Aa} \alpha$ & $8,9 \mathrm{Aa} \alpha$ & $8,3 \mathrm{Aa} \alpha$ \\
\hline & $\mathrm{U}+\mathrm{CC}$ & $8,6 \mathrm{Aa} \alpha$ & $9,3 \mathrm{Aa} \alpha$ & $9,2 \mathrm{Aa} \alpha$ & $9,5 \mathrm{Aa} \alpha$ & $9,0 \mathrm{Aa} \alpha$ & $9,6 \mathrm{Aa} \alpha$ & $8,4 \mathrm{Aa} \alpha$ \\
\hline & IB & $7,9 \mathrm{Ab} \alpha$ & $9,2 \mathrm{Aab} \alpha$ & $9,7 \mathrm{Aa} \alpha$ & $9,5 \mathrm{Aab} \alpha$ & $9,4 \mathrm{Aab} \alpha$ & $8,7 \mathrm{Aab} \alpha$ & $8,4 \mathrm{Aab} \alpha$ \\
\hline \multirow{5}{*}{ M71 } & $\mathrm{T}$ & $9,6 \mathrm{Aa} \alpha$ & $6,9 \mathrm{Ab} \beta$ & $6,6 \mathrm{Ab} \beta$ & $6,5 \mathrm{Ab} \beta$ & $6,3 \mathrm{Bb} \beta$ & $6,6 \mathrm{Ab} \beta$ & $6,3 \mathrm{Ab} \beta$ \\
\hline & $\mathrm{U}$ & $7,1 \mathrm{Ba} \beta$ & $6,3 \mathrm{Aa} \beta$ & $6,7 \mathrm{Aa} \beta$ & $6,8 \mathrm{Aa} \beta$ & $6,6 \mathrm{Ba} \beta$ & $6,2 \mathrm{Aa} \beta$ & $6,6 \mathrm{Aa} \beta$ \\
\hline & $\mathrm{CC}$ & $8,2 \mathrm{Ba} \alpha$ & $7,3 \mathrm{Aab} \beta$ & $7,5 \mathrm{Aab} \beta$ & $7,3 \mathrm{Aab} \beta$ & $6,6 \mathrm{Bab} \beta$ & $6,7 \mathrm{Aab} \beta$ & $6,3 \mathrm{Ab} \beta$ \\
\hline & $\mathrm{U}+\mathrm{CC}$ & $7,1 \mathrm{Ba} \beta$ & $6,8 \mathrm{Aa} \beta$ & $6,6 \mathrm{Aa} \beta$ & $6,5 \mathrm{Aa} \beta$ & $6,3 \mathrm{Ba} \beta$ & $6,2 \mathrm{Aa} \beta$ & $6,3 \mathrm{Aa} \beta$ \\
\hline & IB & 7,7Bab $\alpha$ & $7,3 \mathrm{Aab} \beta$ & $6,7 \mathrm{Ab} \beta$ & $7,0 \mathrm{Ab} \beta$ & $8,5 \mathrm{Aa} \alpha \beta$ & $6,8 \mathrm{Ab} \beta$ & $6,4 \mathrm{Ab} \beta$ \\
\hline
\end{tabular}

Valores seguidos por letras maiúsculas distintas na mesma coluna, dentro de um genótipo, diferem entre si ( $\mathrm{P}<0,05$; efeito aditivo); Valores seguidos por letras minúsculas distintas na mesma linha diferem entre si ( $\mathrm{P}<0,05$; efeito abertura);

Valores seguidos por letras gregas distintas na mesma coluna diferem entre si $(\mathrm{P}<0,05$; efeito de genótipo e aditivos);

${ }^{1} \mathrm{~V} 2$ = V2000; R9 = Rumbosol 91; M7 = M734. CV = 7,236. Teste SNK $(\mathrm{P}<0,05)$.

Quanto ao CC, apesar de apresentar silagens com valores de lignina semelhantes à silagem $\mathrm{T}$ em todos os dias de abertura, mostrou redução durante o processo fermentativo para o genótipo M734, em que foi verificada concentração de $8,2 \%$ de lignina no primeiro dia de abertura e conseqüente redução para $6,3 \%$ no $56^{\circ}$ dia. A adição da $\mathrm{U}+\mathrm{CC}$ não resultou em mudanças nos teores de lignina em todo o período fermentativo observado.

Os teores de lignina permaneceram intactos nas silagens, mesmo com a presença dos aditivos, estando de acordo com Van Soest (1994), o qual afirmou que os teores de lignina permanecem estáveis com o avanço do processo fermentativo. 
As silagens testemunhas apresentaram no dia de abertura 56 valores de $51,0 \%, 49,1 \%$ e $48,9 \%$ de DIVMS para os genótipos M734, V2000 e
Rumbosol 91, respectivamente, não havendo diferença entre eles (Tab. 6). O mesmo foi observado no decorrer do processo fermentativo.

Tabela 6. Valores da digestibilidade in vitro da matéria seca (\% MS) das silagens de girassol testemunha $(\mathrm{T})$, tratadas com $0,5 \%$ de uréia (U), $0,5 \%$ de carbonato de cálcio $(\mathrm{CC}), 0,5 \%$ de uréia associada a $0,5 \%$ de carbonato de cálcio (U+CC) e inoculante bacteriano (IB) nos diferentes dias de abertura

\begin{tabular}{|c|c|c|c|c|c|c|c|c|}
\hline \multirow[b]{2}{*}{ Genótipo } & \multirow[b]{2}{*}{ Trat. } & \multicolumn{7}{|c|}{ Dias de abertura dos silos } \\
\hline & & 1 & 3 & 5 & 7 & 14 & 28 & 56 \\
\hline \multirow{5}{*}{$\mathrm{V} 2^{1}$} & $\mathrm{~T}$ & $45,7 \mathrm{Aab} \alpha$ & $47,1 \mathrm{Aab} \alpha$ & $47,7 \mathrm{Aab} \alpha$ & $41,4 \mathrm{Bb} \beta$ & $45,8 \mathrm{Aab} \alpha$ & $47,3 \mathrm{Aab} \alpha$ & $49,1 \mathrm{Ba} \alpha$ \\
\hline & $\mathrm{U}$ & $48,2 \mathrm{Ab} \alpha$ & $51,0 \mathrm{Ab} \alpha$ & $47,5 \mathrm{Ab} \alpha$ & $45,7 \mathrm{ABb} \alpha$ & $47,2 \mathrm{Ab} \alpha$ & $49,0 \mathrm{Ab} \alpha$ & $55,9 \mathrm{Aa} \alpha$ \\
\hline & $\mathrm{CC}$ & $49,1 \mathrm{Aa} \alpha$ & $52,5 \mathrm{Aa} \alpha$ & $47,9 \mathrm{Aa} \alpha$ & $45,2 \mathrm{ABa} \beta$ & $49,3 \mathrm{Aa \alpha}$ & $48,8 \mathrm{Aa} \alpha$ & $52,5 \mathrm{ABa} \alpha$ \\
\hline & $\mathrm{U}+\mathrm{CC}$ & $51,7 \mathrm{Aa} \alpha$ & $52,3 \mathrm{Aa} \alpha$ & $48,2 \mathrm{Aa} \alpha$ & $47,7 \mathrm{Aba \alpha}$ & $51,5 \mathrm{Aa} \alpha$ & $49,0 \mathrm{Aa} \alpha$ & $49,2 \mathrm{Ba} \alpha$ \\
\hline & IB & $50,4 \mathrm{Aa} \alpha$ & $50,1 \mathrm{Aa} \alpha$ & $48,4 \mathrm{Aa} \alpha$ & $50,5 \mathrm{Aa} \alpha$ & $51,5 \mathrm{Aa} \alpha$ & $48,4 \mathrm{Aa} \alpha$ & $50,0 \mathrm{Ba} \alpha$ \\
\hline \multirow{5}{*}{$\mathrm{R} 9^{1}$} & $\mathrm{~T}$ & $45,8 \mathrm{Aa \alpha}$ & $48,2 \mathrm{Aa} \alpha$ & $48,3 \mathrm{Aa} \alpha$ & $45,3 \mathrm{Aa} \alpha \beta$ & $47,4 \mathrm{Aa} \alpha$ & $46,3 \mathrm{Aa} \alpha$ & $48,9 \mathrm{Aa} \alpha$ \\
\hline & $\mathrm{U}$ & 49,9Аа $\alpha$ & $51,0 \mathrm{Aa} \alpha$ & $47,6 \mathrm{Aa} \alpha$ & $47,6 \mathrm{Aa} \alpha$ & $46,4 \mathrm{Aa} \alpha$ & $46,1 \mathrm{Aa} \alpha \alpha$ & $47,4 \mathrm{Aa} \beta$ \\
\hline & $\mathrm{CC}$ & $47,9 \mathrm{Aa} \alpha$ & $46,3 \mathrm{Aa} \beta$ & $46,8 \mathrm{Aa} \alpha$ & $45,8 \mathrm{Aa} \beta$ & $45,3 \mathrm{Aa} \alpha$ & $45,8 \mathrm{Aa} \alpha$ & $45,6 \mathrm{Aa} \beta$ \\
\hline & $\mathrm{U}+\mathrm{CC}$ & $36,5 \mathrm{Bb} \beta$ & $47,0 \mathrm{Aa} \alpha$ & $44,3 \mathrm{Aa} \alpha$ & $45,4 \mathrm{Aa} \alpha$ & $45,2 \mathrm{Aa} \beta$ & $45,8 \mathrm{Aa} \alpha$ & $48,5 \mathrm{Aa} \alpha$ \\
\hline & IB & $49,1 \mathrm{Aa} \alpha$ & $49,1 \mathrm{Aa} \alpha$ & $45,7 \mathrm{Aa} \alpha$ & $46,3 \mathrm{Aa} \alpha$ & 49,1Aa $\alpha$ & $46,6 \mathrm{Aa} \alpha$ & $51,8 \mathrm{Aa} \alpha$ \\
\hline \multirow{5}{*}{$\mathrm{M} 7^{1}$} & $\mathrm{~T}$ & $42,5 \mathrm{Bb} \alpha$ & 49,9Aa $\alpha$ & $48,1 \mathrm{Aab} \alpha$ & $50,0 \mathrm{Aa} \alpha$ & $51,2 \mathrm{Aa} \alpha$ & $48,3 \mathrm{Aab} \alpha$ & $51,0 \mathrm{Aa} \alpha$ \\
\hline & $\mathrm{U}$ & $51,0 \mathrm{Aa} \alpha$ & $51,2 \mathrm{Aa} \alpha$ & $50,0 \mathrm{Aa} \alpha$ & $50,4 \mathrm{Aa} \alpha$ & $46,2 \mathrm{Aa} \alpha$ & $48,1 \mathrm{Aa} \alpha$ & $50,1 \mathrm{Aa} \beta$ \\
\hline & $\mathrm{CC}$ & $50,7 \mathrm{Aa} \alpha$ & $49,5 \mathrm{Aa} \alpha \beta$ & $50,7 \mathrm{Aa} \alpha$ & $51,4 \mathrm{Aa} \alpha$ & $46,3 \mathrm{Aa} \alpha$ & $48,8 \mathrm{Aa} \alpha$ & $49,2 \mathrm{ABa} \alpha \beta$ \\
\hline & $\mathrm{U}+\mathrm{CC}$ & $52,0 \mathrm{Aa} \alpha$ & $50,0 \mathrm{Aab} \alpha$ & $50,0 \mathrm{Aab} \alpha$ & $51,1 \mathrm{Aa} \alpha$ & $48,8 \mathrm{Aab} \alpha \beta$ & $50,1 \mathrm{Aab} \alpha$ & $43,7 \mathrm{Bb} \alpha$ \\
\hline & IB & $49,2 \mathrm{Aa} \alpha$ & $47,5 \mathrm{Aa} \alpha$ & $49,5 \mathrm{Aa} \alpha$ & 48,9Aa $\alpha$ & $47,9 \mathrm{Aa} \alpha$ & $49,3 \mathrm{Aa} \alpha$ & $47,1 \mathrm{ABa} \alpha$ \\
\hline
\end{tabular}

Valores seguidos por letras maiúsculas distintas na mesma coluna, dentro de um genótipo, diferem entre si ( $\mathrm{P}<0,05$; efeito aditivo); Valores seguidos por letras minúsculas distintas na mesma linha diferem entre si ( $\mathrm{P}<0,05$; efeito abertura);

Valores seguidos por letras gregas distintas na mesma coluna diferem entre si $(\mathrm{P}<0,05$; efeito de genótipo e aditivos);

${ }^{1} \mathrm{~V} 2=\mathrm{V} 2000 ; \mathrm{R} 9=$ Rumbosol 91; M7 = M734. CV = 5,167. Teste SNK $(\mathrm{P}<0,05)$.

O tratamento com U não proporcionou alteração na DIVMS no decorrer do processo fermentativo para as silagens de girassol do Rumbosol $91 \mathrm{e}$ M734, as quais variaram de $46,1 \%$ a $51,2 \%$ e $46,2 \%$ a $51,2 \%$, respectivamente. Quanto ao V2000, houve aumento significativo para silagem no dia 56 , cujo valor foi de $55,9 \%$. De acordo com Vilela (1998), a amônia liberada da hidrólise da uréia pode causar quebra de ligações esteres entre lignina, celulose e hemiceluloses aumentando a DIVMS das silagens. Schingoethe et al. (1980) observaram aumento da DIVMS em silagem de girassol tratada com uréia em relação à silagem controle $(62,0 \%$ vs 59,6\%). Scheffer de Rojas (1976) e Gonçalves et al. (1998) trabalharam com silagens de milho tratadas com $0,5 \%$ de uréia, e não encontraram efeito da uréia sobre a característica. A não resposta quanto à utilização da uréia nas silagens dos genótipos em relação às silagens testemunhas, pode estar relacionada com a elevada concentração de proteína presente nas plantas no momento da ensilagem.

O uso do CC nas silagens dos três genótipos não causou mudanças nos teores da DIVMS quando comparadas às silagens $\mathrm{T}$ em praticamente todos os dias de abertura, confirmando os resultados de Scheffer de Rojas (1976), que utilizaram a mesma proporção desse aditivo em silagem de milho. O tratamento com $\mathrm{U}+\mathrm{CC}$ nas silagens, com exceção do valor de $36,5 \%$ encontrado no primeiro dia de abertura para o Rumbosol $91 \mathrm{e}$ $43,7 \%$ no dia 56 para o M734, os quais foram diferentes das silagens $\mathrm{T}$, não resultou em alterações no decorrer do processo fermentativo.

A DIVMS das silagens tratadas com IB foi semelhante à das silagens testemunhas em todos os dias de abertura. Resultado semelhante foi observado por Meeske et al. (1993) que, ao trabalharem com silagens de sorgo, encontraram $64,7 \%$ e $60,7 \%$ de DIVMS nas silagens controle e com inoculante, respectivamente, enquanto Hunt et al. (1993) observaram uma diminuição na DIVMS da silagem de milho inoculada $(67,9 \%)$ quando comparada com at silagem controle $(69 \%)$.

Houve correlação negativa $(\mathrm{P}<0,01 \%)$ entre a DIVMS e todos os constituintes da fração fibrosa, FDN $(r=-0,51)$, FDA $(r=-0,50)$, 
hemiceluloses $(r=-0,25)$, celulose $(r=-0,48)$ e lignina $(r=-0,41)$, indicando que a digestibilidade da silagem é comprometida com o aumento da concentração fibrosa na forragem.

\section{CONCLUSÕES}

Os aditivos utilizados neste experimento não promoveram alterações nas características avaliadas, indicando que a recomendação desses produtos para silagens de girassol, provavelmente, não resultem em melhoria de qualidade.Com base na digestibilidade in vitro da matéria seca, os genótipos teriam aproveitamentos semelhantes pelos animais.

\section{REFERÊNCIAS BIBLIOGRÁFICAS}

BARTH, K.M.; CORRICK, J.A.; SHUMWAY, P.E. et al. Effects of level urea and of urea plus limestone on $\mathrm{N}$ metabolism of corn silage-based rations by cattle. J. Anim. Sci., v.38, p.687-692, 1974.

FREIRE, E.M. Padrão de fermentação das silagens de cinco hibridos de girassol (Helianthus annuus L.). 2001. 44p. Dissertação (Mestrado) - Escola de Veterinária, Universidade Federal de Minas Gerais, Belo Horizonte.

GONÇALVES, L.C.; BORGES, A.L.C.C.; RODRIGUEZ, N.M. et al. Valor nutritivo de silagens de milho puras, ou adicionadas de uréia pura ou com carbonato de cálcio, e do rolão de milho. I - Consumo e digestibilidade aparente da matéria seca, da proteína e balanço de nitrogênio. Arq .Bras .Med. Vet. Zootec., v.50, p.309-315, 1998.

HIGGINBOTHAM, G.E.; MUELLER, S.C.; BOLSEN, K.K. et al. Effects of inoculants containing propionic acid bacteria on fermentation and aerobic stability of corn silage. J. Dairy Sci., v.81, p.2185-2192, 1998.

HUBER, J.T.; THOMAS, J.W.; EMERY, R.S. Response of lactating cows fed urea-treated corn silage harvested at varying stages of maturity. $J$. Dairy Sci., v.51, p.1806-1810, 1968.

HUNT, C.W.; KEZAR, W.; HINMAN, D.D. et al. Effects of hybrid and ensiling with and without a microbial inoculant on the nutritional characteristics of whole-plant corn. J. Anim. Sci., v.71, p.38-43, 1993.

ISEPON, O.J.; BERGAMASCHINE, A.F.; GUATURA, A.S. et al. Qualidade da silagem de girassol colhido em duas idades, na presença ou ausência de inoculante microbiano. In: REUNIÃO ANUAL DA SOCIEDADE BRASILEIRA DE ZOOTECNIA, 37., 2000, Viçosa. Anais... Viçosa, SBZ, 2000. (Resumo).

McDONALD, P.; HENDERSON, A.R.; HERON, S. The biochemistry of silage. 2.ed. Marlow: Chalcombe Publications, 1991. 340p.

MEESKE, R.; ASHBELL, G.; WEINBERG, Z.G. et al. Ensiling forage sorghum at two stages of maturity with the addition of lactic acid bacterial inoculants. Anim. Feed Sci. Techonol., v.43, p.165-175, 1993.

MUCK, R.E. Factors influencing silage quality and their implications for management. J. Dairy Sci., v.71, p.2992-3002, 1988.

NOGUERA, J.R.R. Qualidade das silagens de quatro cultivares de girassol (Helianthus annuus L.) ensilados com diferentes proporções da planta. 2000. 63p. Dissertação (Mestrado) Escola de Veterinária, Universidade Federal de Minas Gerais, Belo Horizonte.

PIRES, A.J.V.; GARCIA, R.; SILVA, F.F. et al. Qualidade de silagens de sorgo (Sorghum bicolor L. Moench) tratadas com uréia e/ou sulfeto de sódio. In: REUNIÃO ANUAL DA SOCIEDADE BRASILEIRA DE ZOOTECNIA, 35., 1998, Botucatu. Anais... Botucatu, SBZ, 1998. (Resumo).

SCHEFFER DE ROJAS, S.A.S. Efeito de aditivos e do momento de vedação na qualidade da silagem de milho em condições de laboratório. 1976. 83p. Tese (Mestrado).

SCHINGOETHE， D.J.; SKYBERG， E.W.; ROOK, J.A. Chemical composition of sunflower silage as influenced by additions of urea, dried whey and sodium hydroxide. J. Anim. Sci., v.50, p.625-629, 1980.

SILVA, A.W.L.; MACEDO, A.F.; MIGUELLUTI, D.J. et al. Efeito do uso de inoculante bacteriano e de diferentes proporções de grãos na massa sobre a composição bromatológica da silagem de milho. In: REUNIÃO ANUAL DA SOCIEDADE BRASILEIRA DE ZOOTECNIA, 34., 1997, Juiz 
de Fora. Anais... Juiz de Fora, SBZ, 1997. p.170172. (Resumo).

SISTEMA de análise estatísticas e genéticas SAEG. Versão 7.1. Viçosa, MG. UFV, 1997. p.150.

SOUZA, D.B. Girassol - Uma nova opção para silagem. Gado Holandês, n.472, p.6-10, 1998.

TILLEY, J.M.A.; TERRY, R.A. A two-stage technique for the in vitro digestion of forage crops. J. Br. Grassl. Soc., v.18, p.104-111, 1963.

TOMICH, T.R.; RODRIGUES, J.A.S.; GONÇALVES, L.C. et al. Potencial forrageiro de cultivares de girassol produzidos na safrinha para ensilagem. Arq. Bras. Med. Vet. Zootec., v.55, p.756-762, 2003.

VAN SOEST, P.J.; ROBERTSON, J.B.; LEWIS, B.A. Methods for dietary fiber, neutral detergent fiber, and nonstarch polysaccharides in relation to animal nutrition. J. Dairy Sci., v.74, p.35833597, 1991.

VAN SOEST, P.J. Nutritional ecology of the ruminant. 2.ed. Ithaca, New York: Cornell University, 1994. 476p.

VIEIRA, F.A.P. Qualidade de silagens de sorgo (Sorghum bicolor L, Moench) com aditivos. 2001. 49f. Dissertação (Mestrado) - Escola de Veterinária, Universidade Federal de Minas Gerais, Belo Horizonte.

VIEIRA, F.A.P.; BORGES, I.; STEHLING, C.A.V. et al. Qualidade de silagens de sorgo com aditivo. Arq. Bras. Med. Vet. Zootec., v.56, p.764-772, 2004.

VILELA, D. Aditivos para silagens de plantas de clima tropical. In: REUNIÃO ANUAL DA SOCIEDADE BRASILEIRA DE ZOOTECNIA, 35., 1998, Botucatu. Anais... São Paulo, SBZ, 1998. p.73-108. (Resumo). 